\title{
Local Polynomially Convex Hulls at Degenerated CR Singularities of Surfaces in $\mathbb{C}^{2}$
}

\section{JAN WIEGERINCK}

\begin{abstract}
The local polynomially convex hull of a surface at isolated degenerated CR singularities is studied. It is shown that under suitable pseudoconvexity conditions at points of positive index, the surface is not locally polynomially convex and contains analytic discs with boundary in the surface. Contrary to the well-known case of elliptic points, it may happen that the boundary of such discs passes through the CR singularity. This may also occur at a point of negative index. For points of index $>1$, we give sufficient conditions for the hull to contain a $\mathbb{C}^{2}$-open neighborhood of the $\mathrm{CR}$ singularity.
\end{abstract}

1. Introduction. Recall that for a compact set $K \subset \mathbb{C}^{n}$ the polynomially convex hull is defined as

$$
\hat{K}=\left\{z \in \mathbb{C}^{n}: \forall P \in \mathbb{C}[z],|P(z)| \leq\|P\|_{K}\right\} .
$$

Here $\|\cdot\|_{K}$ denotes the supremum norm on $K$ and $\mathbb{C}[z]$ the polynomials on $\mathbb{C}^{n}$. We say that a closed set $K$ is locally polynomially convex at $p$ if, for some $\varepsilon>0$, the set $(\bar{B}(p, \varepsilon) \cap K)^{\wedge}$ equals $\bar{B}(p, \varepsilon) \cap K$. An analytic disc in $\mathbb{C}^{n}$ is defined as a non-constant continuous map from the closed unit disc in $\mathbb{C}$ to $\mathbb{C}^{n}$ which is holomorphic on the interior.

In this paper we intend to study locally the polynomially convex hulls of surfaces $\Gamma$ in $\mathbb{C}^{2}$, that is the sets $(\bar{B}(p, \varepsilon) \cap K)^{\wedge}$ for small $\varepsilon$. E. Bishop suggested in $[\mathrm{B}]$ that this is a topic worth studying and he obtained the first, by now well known, results. At a totally real point $p$ on the surface $\Gamma$, that is, the tangent space $T_{p}(\Gamma)$ is not a complex line, $\Gamma$ is locally polynomially convex. Points that are not totally real will be called $\mathrm{CR}$ singularities or exceptional points. The polynomially convex hull at isolated CR singularities is fairly well understood when the order of contact of the surface with the tangent plane equals 2 . Then a local holomorphic change of coordinates will translate the point to the origin and 
the surface $\Gamma$ will locally be a graph of the form $w=|z|^{2}+\lambda\left(z^{2}+\bar{z}^{2}\right)+O\left(|z|^{3}\right)$, $\lambda>0$, or $w=z^{2}+\bar{z}^{2}+O\left(|z|^{3}\right)$. Bishop calls a point elliptic if $\lambda<\frac{1}{2}$, parabolic if $\lambda=\frac{1}{2}$ and hyperbolic in the remaining cases. He shows that at an elliptic point there exists a one parameter family of analytic discs with boundary in $\Gamma$ belonging to the polynomially convex hull of $\Gamma \cap \bar{B}(0, \varepsilon)$; the elliptic point is not in the boundary of any of these discs. If there are no higher order terms present, the graph is contained in $\mathbb{C} \times \mathbb{R}$ and the discs are just of the form $w=t, t>0$. At a hyperbolic point, $\Gamma$ is locally polynomially convex, cf. [FS]. Subsequently, these results were refined and the needed amount of smoothness was lowered, cf. $[\mathrm{KW}, \mathrm{HT}]$. At a parabolic point, the local behavior of the polynomially convex hull depends on the higher order terms and is much less understood.

Bishop introduced the term exceptionally exceptional points for CR singularities which are exceptional but not hyperbolic or elliptic in the above sense, e.g., the order of contact of the complex line tangent at $p$ may be $>2$. We will also call such points degenerated CR singularities, which is more informative. Bishop's terminology may be justified by Thom's transversality theorem, and the fact that the complex lines in $\mathbb{C}^{2}$ are of codimension 2 in the Grassmann manifold of 2-planes in $\mathbb{C}^{2}$, cf. [GG]; e.g., any embedded sphere in $\mathbb{C}^{2}$ has at least 2 exceptional points, but spheres without exceptionally exceptional points are dense.

For an isolated CR singularity an index, which is essentially the Maslov index, see $[\mathrm{M}]$, may be introduced cf. $[\mathrm{F} 1]$, or $[\mathrm{G}]$ for the higher dimensional case. Having a positive index will serve as our substitute for being an elliptic point. Indeed, elliptic points have index 1, hyperbolic points have index -1 . Moreover, Forstneric's result, quoted in Section 2, will give that once one disc is present with boundary passing around a point of positive index, then there are many. Motivated by examples like $w=z\left(|z|^{2}+\lambda\left(z^{2}+\bar{z}^{2}\right)\right), \lambda<\frac{1}{2}$, where there are discs of the form $w=t z$ for $z$ in a suitable domain, one might hope for the presence of discs with the same nice behavior as in the elliptic case at isolated degenerated CR singularities. This will turn out to be too optimistic. The boundary of an analytic disc may well pass through a CR singularity of positive index. Observe that at an elliptic point a surface is locally contained in the boundary of a strictly pseudoconvex domain. In general this is a condition that has to be imposed. Also, in the elliptic case this surface is "very close" to the graph of the function identically equal to 0 on the boundary of a convex domain in $\mathbb{C} \times \mathbb{R}$ (that is the use of the particular coordinate transform). In fact in the model case, without higher order terms, we are dealing with the graph of the zero function above $\operatorname{Re} w=|z|^{2}+\lambda\left(z^{2}+\bar{z}^{2}\right) \subset \mathbb{C} \times \mathbb{R}$. This is very helpful in the classical case, but it will no longer be at our disposal.

We will study the polynomially convex hull of surfaces at isolated degenerated CR singularities. It is shown that under suitable pseudoconvexity conditions, a positive index leads to a local polynomially convex hull which contains 
analytic discs having their boundary in the surface close to the CR singularity. For graphs of homogeneous functions we will give necessary and sufficient conditions that assure that these boundaries don't pass through the CR singularity. The pseudoconvexity conditions will automatically be satisfied for isolated CR singularities of positive index at which the tangent plane has order of contact $k \leq 3$, with the surface. If $k>3$, this is not true in general.

Thus, as a special case we find that at a parabolic point of positive index 1 , the polynomially convex hull consists locally of a one parameter family of analytic discs.

If the index of a parabolic point $p$ is negative, Jöricke $[\mathrm{J}]$ has shown very recently that the surface is locally polynomially convex at $p$. At certain special types of isolated degenerated CR singularities of negative index, Harris found local polynomial convexity, cf. $[\mathrm{H}]$. Forstneric [F2] gave an example of a holomorphically convex smooth disc with an index 0 singularity with a local polynomially convex hull containing a discrete but not continuous family of discs.

In Section 4 we will give 3 examples:

1. A graph of a homogeneous polynomial of degree 3 which has index 2 at the origin and is such that the local hull contains a one parameter family of discs with boundary passing through the origin.

2. A graph of a homogeneous polynomial of degree 8 which has index 1 at the origin and has the property that the local hull consists of a one-parameter family of analytic discs with boundary passing through the origin.

3. A graph of a homogeneous polynomial of degree 6 which has index -1 at the origin and has the property that the local hull consists of a one-parameter family of analytic discs with boundary passing through the origin.

In the next section we will recall known results and derive some fairly straightforward consequences that will be needed in Section 3 .

2. Analytic discs in Levi-flat hypersurfaces. In this section we will recall some pertinent results dealing with analytic discs with boundary in a smooth surface, in particular a topological sphere, in the boundary of a strictly pseudoconvex domain. In most of this section the discs belong to a one-parameter family that forms a hypersurface in $\mathbb{C}^{2}$. Such a hypersurface will be Levi-flat, that is, locally the complement is pseudoconvex. If the hypersurface is $C^{2}$, this just means that the Levi-form of the hypersurface vanishes. Almost everything in this section is already known; we include it for convenience of the reader.

We will need the main theorem from a paper of E. M. Chirka and N. V. Shcherbina, cf. [CSh]. Consider sets in $\mathbb{C} \times \mathbb{R}$ with coordinates $(z, u)$ and view $\mathbb{C} \times \mathbb{R}$ as the subspace $\{v=0\}$ of $\mathbb{C}^{2}=\mathbb{C} \times \mathbb{R} \times i \mathbb{R}$ with coordinates $(z, w)=$ $(z, u+i v)$. Graphs of functions $(i \times) v(z, u)$ on subsets of $\mathbb{C} \times \mathbb{R}$ will be denoted by $\Gamma_{v}$. 
Theorem 2.1 (Chirka-Shcherbina). Let $\Omega$ be a bounded domain in $\mathbb{C} \times \mathbb{R}$ of the form $h^{-}(z)<u<h^{+}(z)$, where $h^{-}$and $-h^{+}$are Hölder continuous subharmonic, but nowhere harmonic functions on a closed domain $\overline{\mathcal{G}} \subset \mathbb{C}$ with the property that $h^{+}=h^{-}$on the boundary of $\mathcal{G}$. Assume that $\Omega$ is homeomorphic to a 3-ball. Let $\varphi \in C(\partial \Omega)$. Then there exists $\Phi \in C(\bar{\Omega})$ such that the polynomially convex hull $\hat{\Gamma}_{\varphi}$ equals $\Gamma_{\Phi}$ and the following properties hold:

(i) The set $\Gamma_{\Phi} \backslash \Gamma_{\varphi}$ is the union of a disjoint family of analytic discs $D_{\alpha}$.

(ii) For each $\alpha$ there exist a simply-connected domain $G_{\alpha} \subset \mathcal{G}$ and a holomorphic function $w=f_{\alpha}(z)$ on $G_{\alpha}$ such that $D_{\alpha}=\left\{\left(z, f_{\alpha}(z)\right): z \in G_{\alpha}\right\}$.

(iii) Each $f_{\alpha}$ admits a continuous extension $f_{\alpha}^{*}$ to $\bar{G}_{\alpha}$ and $\partial D_{\alpha}=\left\{\left(z, f_{\alpha}^{*}(z)\right)\right.$ : $\left.z \in \partial G_{\alpha}\right\}$ is contained in $\Gamma_{\varphi}$.

(iv) For every $\alpha$, the set $\mathcal{G} \backslash \bar{G}_{\alpha}$ contains no connected components that are relatively compact in $\mathcal{G}$.

To derive additional properties, we recall how in [CSh] and basically also in [Sh], the function $\Phi$ is constructed. Let $\mathcal{F}_{\varphi}$ be the set of all lower semicontinuous functions $F$ on $\bar{\Omega}$ such that $F>\varphi$ on $\partial \Omega$, that have the additional properties that for $P \in \partial \Omega, \liminf _{\Omega \ni P^{\prime} \rightarrow P} F\left(P^{\prime}\right)=F(P)$ and that the domain $(\Omega \times i \mathbb{R}) \cap$ $\{v<F(z, u)\}$ is pseudoconvex. Since $\varphi$ is bounded, $\mathcal{F}$ is non-empty. The function $\Phi$ in Theorem 2.1 is obtained as follows.

$$
\Phi_{0}(P)=\inf \left\{F(P): F \in \mathcal{F}_{\varphi}\right\} \quad \text { and } \quad \Phi(P)=\liminf _{P^{\prime} \rightarrow P} \Phi_{0}\left(P^{\prime}\right)
$$

This leads to the following corollary of the construction.

Corollary 2.2. $\quad$ Suppose that $\varphi_{1} \geq \varphi_{2}$ on $\partial \Omega$. Then $\Phi_{1} \geq \Phi_{2}$ on $\bar{\Omega}$. Moreover, if $f_{j}$ are holomorphic functions on domains $D_{j}$ which define analytic discs in $\Gamma_{\Phi_{j}} \backslash \Gamma_{\varphi_{j}}(j=1,2)$, then (the interiors of) these discs are disjoint or the discs coincide.

Proof. The first part is clear because for $\Phi_{1}$ we take the infimum over a smaller set.

For the second part, observe that if $\varphi^{\prime}=\varphi_{2}+\varepsilon$, then $\Phi^{\prime}=\Phi_{2}+\varepsilon$. Hence, the first part implies that if $\varphi_{1}>\varphi_{2}$, then $\Phi_{1}>\Phi_{2}$. Suppose that the discs defined by $f_{1}$ and $f_{2}$ have non-empty intersection. Then either $f_{1}$ and $f_{2}$ are identical on an open set and hence the discs are the same, or the function $f_{1}-f_{2}$ would have an isolated zero on $D_{1} \cap D_{2}$, but by Rouché's theorem $f_{1}+i \varepsilon-f_{2}$ would also have a zero for small positive $\varepsilon$. This would contradict that $\Phi_{1}+\varepsilon>$ $\Phi_{2}$. 
Corollary 2.3. The "harmonic discs" $\left(z, \operatorname{Re} f_{\alpha}(z)\right), z \in G_{\alpha}$ foliate $\Omega$. Thus,

$$
\exists z \in G_{\alpha} \cap G_{\beta}: \operatorname{Re} f_{\alpha}(z)>\operatorname{Re} f_{\beta}(z) \Rightarrow \operatorname{Re} f_{\alpha}>\operatorname{Re} f_{\beta} \text { on } G_{\alpha} \cap G_{\beta}
$$

and either $G_{\alpha} \cap G_{\beta}$ is empty or one is contained in the other. Moreover, if the harmonic discs above $G_{\alpha}$ and $G_{\beta}$ have their boundaries in $u=h^{-}(z)$, then $G_{\beta} \subset G_{\alpha}$ implies $\operatorname{Re} f_{\alpha}>\operatorname{Re} f_{\beta}$ on $G_{\beta}$.

Proof. Obvious; the last statement follows from the maximum principle.

\section{Remarks 2.4.}

1. The conditions on $\Omega$ ensure that $\Omega \times i \mathbb{R}$ is a pseudoconvex domain in $\mathbb{C}^{2}$. Shcherbina originally obtained a theorem like 2.1 for convex domains $\Omega$, cf. [Sh].

2. Concerning the boundary behaviour of the discs $D_{\alpha}$, it follows from work of Chirka [C] that $f_{\alpha}$ belongs to $C^{k-\varepsilon}$ at any boundary point of $D_{\alpha}$ that is mapped to a totally real point of $\Gamma_{\varphi}$ at which $\Gamma_{\varphi}$ is $C^{k}$.

3. Theorem 2.1 implies, of course, that $\Gamma_{\Phi}$ is a Levi-flat hypersurface above $\Omega$.

We take the following definition from Forstneric's paper [F1].

Definition 2.5. Let $T$ be the unit circle in $\mathbb{C}$ and let $\gamma=\{F(\zeta), \zeta \in T\}$ be a simple closed curve in a $C^{1}$ surface $\Gamma \subset \mathbb{C}^{2}$ which is totally real at the points of $\gamma$. Assume that $\Gamma$ is orientable (on a neighborhood of $\gamma$ ). Then the Index $I(\gamma)$ of $\gamma$ is the winding number of $\operatorname{det}(X(\zeta), Y(\zeta))$ along $T$, where $\{X(\zeta), Y(\zeta)\}$ is a basis for the tangent space $T_{F(\zeta)}(\Gamma)$, that varies smoothly with $\zeta$. The index of an analytic disc with boundary in $\Gamma$ is defined as the index of its boundary.

Let $\Gamma \subset \mathbb{C}^{2}$ be a totally real surface except for an isolated CR-singularity at $p$. Then the Index of $p$ is the index of a simple closed curve around $p$.

One can show as in [F1], that the index is independent of the choice of $X$ and $Y$ and of the parametrization of $\gamma$ and that the index of an isolated CR-singularity is independent of the choice of the curve around it. In [F1], see also $[G]$, the index is defined in a more general setting, and the following computational lemma is given.

Lemma 2.6. Let $\Gamma$ be of the form $w=\varphi(z, \bar{z})$ and let $\gamma \subset \Gamma$ be given by $w=\varphi(z, \bar{z}), z \in \gamma_{0}$ a simple closed curve in $\mathbb{C}$. Then

$$
I(\gamma)=\text { winding number of } \frac{\partial \varphi}{\partial \bar{z}} \text { along } \gamma
$$

We will use the following form of Theorems 1-3 of Forstneric, cf. [F1]. 
Theorem 2.7 (Forstneric). Suppose that $\gamma \subset \Gamma$ is the boundary of an immersed analytic disc $F: \bar{D} \rightarrow \mathbb{C}^{2}$ in a totally real surface $\Gamma$ of class $C^{2}$. If the index of $\gamma$ equals $k>0$, then $F$ belongs to a $2 k-1$ parameter family of analytic discs with boundary in $\Gamma$. Moreover, for any surface $\Gamma^{*}$ sufficiently $C^{2}$ close to $\Gamma^{*}$ there exists such a family with boundaries in $\Gamma^{*}$.

In case $k=1$ and if $\Gamma$ is $C^{3}$, the discs form a foliation of a Levi-flat hypersurface.

In case $k>1$, their union contains an open ball.

If the index is less than 1, the disc can not belong to a continuous family of discs with boundary in $\Gamma$.

Next we need to understand what the foliation of the polynomially convex hull of a graph over a generic smooth 3 -sphere in the boundary of a pseudoconvex domain looks like. The existence of a foliation is contained in Theorem 2.1, but the precise form was studied already much earlier by Bedford and Gaveau, [BG], Bedford and Klingenberg [BK] and most recently Kruzhilin [K]. For our purposes Kruzhilin's version is most useful.

Theorem 2.8 (Kruzhilin). Let $U$ be a strictly pseudoconvex domain in $\mathbb{C}^{2}$, and $S$ a $C^{6}$ smooth two dimensional sphere embedded into $\partial U$ and totally real outside a finite subset of $K$ hyperbolic and $K+2$ elliptic points. Then there exist:

1. A closed smooth domain $W \subset \mathbb{R}^{3}\left(x_{1}, x_{2}, x_{3}\right)$ with boundary diffeomorphic to $S$ such that:

(a) the level sets of the function $x_{3}$ on $W$ are unions of finite numbers of topological discs,

(b) $x_{3}$ is a Morse function on $\partial W$ with $K+2$ extreme points and $K$ saddle points;

2. A continuous injective map $\Psi: W \rightarrow U$, taking $\partial W$ to $S$, the extreme points (resp. saddle points) of $x_{3}$ to the elliptic (resp. hyperbolic) points of $S$ and the connected components of the level sets $\left\{x \in W: x_{3}=\right.$ const $\}$ to smooth holomorphic discs.

The set $\Psi(W)$ is the intersection of pseudoconvex neighborhoods of $S$ as well as the hull of $S$ with respect to the algebra $A(U)$ of continuous functions on $\bar{U}$ that are holomorphic on $U$.

Corollary 2.9. Let $S$ be as above and let $I$ be a smooth arc in $S$ that avoids the CR-singularities. Suppose that I meets the boundaries of the analytic discs transversally, then it meets every analytic disc at most once.

Proof. Pull $I$ back via $\Psi$. The result is an arc in the boundary of $W$. If $x_{3}=c$ has two points in common with this arc, then $x_{3}$ would have a stationary point in between, contradicting the transversality. 
Corollary 2.10. Let $\gamma$ be the boundary of a simply connected domain $G \subset S$ that has the property that it contains strictly less hyperbolic than elliptic points. Then there exists an analytic disc with the boundary contained in $\bar{G}$ that meets the curve $\gamma$.

Proof. Again pulling everything back to $W$, we can assume that $\gamma$ and $G$ are on $\partial W$ and we have to show that some level set of $x_{3}$ is contained in $\bar{G}$ and meets $\gamma$. Notice that a simple closed curve, which is (part of) a level set of $x_{3}$ on $\partial W$, has index one and so the number of extrema for $x_{3}$ that $\gamma$ encloses equals the number of saddles +1 . Moreover, a component of a level set passing through $p$ saddles divides $\partial W$ into $p+2$ disjoint components all of which have the number of extremes on it equal to the number of saddles +1 .

We proceed by induction on the number of extremes for $x_{3}$ inside $G$. If there is only 1 assume there is a (local) maximum for $x_{3}$ with value $M$. The level sets $x_{3}=M-t$ will expand if $t$ increases from 0 until they meet $\gamma$.

Now assume that the result has been proved for simply connected domains with $n$ extrema for $x_{3}$ inside, and that $G$ contains $n+1$ extremes. Start with the level sets of $x_{3}$ at an extreme point. Again we may assume there is a local maximum and $x_{3}=M$. The level sets $x_{3}=M-t$ may have several components inside $G$, one of them being a Jordan curve. This will expand as $t$ increases. Either this curve will expand to hit $\gamma$ or it will hit a component $C$ of a level set of $x_{3}$ passing through a saddle. If $C$ lies compactly in $G$, we can further increase $t$ and continue as before. Else, $C$ must meet $\partial G$. If $C \subset \bar{G}$, the boundary of one of the components of $G \backslash C$ is a subset of $C$ meeting $\partial G$ and this component determines the disc we are looking for. If $C$ leaves $\bar{G}$, assume $C$ has $p$ saddles inside $G$. $G \backslash C$ has then $p+2$ simply connected components, of which at least 2 have part of $\gamma$ in their boundary. The assumption implies that these latter components together must contain more extrema than saddles inside $G$. Pick a component with this property. The induction hypothesis can be applied to these domains and it follows that this component contains a level set of $x_{3}$ which meets its boundary. This level set can not meet $C$ because $C$ itself is a component of a level set, hence it meets $\gamma$.

As before, let $U$ be a strictly pseudoconvex domain and $\Gamma$ a totally real surface lying in the boundary of $U$. At a point $x \in \Gamma$, the tangent space $T_{x}(\Gamma)$ forms a two-dimensional subspace of $T_{x}(\partial U)$, which intersects the complex tangent space $T_{x}^{\mathbb{C}}(\partial U)$ in a real line, because $\Gamma$ is totally real. Thus one obtains a smoothly varying field of directions $\chi$ on a neighborhood of $x \in \Gamma$.

Let $F: \bar{D} \rightarrow \bar{U}$ be an analytic disc with boundary in $\Gamma$ passing through $x$. Then the boundary of $F$ is transversal to $\chi$ at $x$. We call integral curves of $\chi=\chi_{\Gamma}$ the forbidden directions. In fact the minimal angle that the boundary of an analytic disc through $x$ can make with $\chi(x)$ is completely determined by the geometry of $\Gamma$ and $U$. This was already observed by Bedford and Gaveau [BG, Section 6]. 
Lemma 2.11. Suppose that $\Gamma$ is given as the graph of a function $g \in$ $C(\partial \Omega)$ in the boundary of a pseudoconvex domain $U=\Omega \times i \mathbb{R}$, where $\Omega \subset \mathbb{C} \times \mathbb{R}$ is bounded and admits an exhaustion function $\rho=\rho(z, u)$ that is plurisubharmonic as a function on $\Omega \times i \mathbb{R}$. Suppose that $\left(z_{0}, u_{0}\right) \in \partial \Omega$ and that $g$ is smooth and $\Omega \times i \mathbb{R}$ is strictly pseudoconvex above a neighborhood of $\left(z_{0}, u_{0}\right)$. There exists a constant $C>0$ depending on the sup norm of $g$ on $\partial \Omega$ and on the $C^{2}$ norm of $g$ and on the Levi form of $\partial \Omega$ both in an arbitrarily small neighborhood $V$ of $\left(z_{0}, u_{0}\right)$, such that if $F=\left(F_{1}, F_{2}\right): \bar{D} \rightarrow \bar{U}$ is an arbitrary analytic disc of which the boundary $F(T)$ satisfies

$$
F(T) \subset \Gamma \quad \text { and } \quad\left(z_{0}, u_{0}+i g\left(z_{0}, u_{0}\right)\right) \in F(T),
$$

then $F(T)$ meets $\chi\left(z_{0}, u_{0}+i g\left(z_{0}, u_{0}\right)\right)$ at an angle at least $C$.

Proof. The proof is as in [BG] as soon as we can obtain functions $\rho_{0}, \rho_{1}$ on $\bar{\Omega}$, depending on $g$ and $\Omega$ as in the statement of the Lemma, which are equal to $g$ on a neighborhood of $\left(z_{0}, u_{0}\right)$ in $\partial \Omega$, and the rest of $\partial \Omega$ satisfy $F(T) \subset\left\{\rho_{0}(z, u) \leq\right.$ $\left.v \leq-\rho_{1}(z, u),(z, u) \in \partial \Omega\right\}$ and are such that the domains $\left\{\rho_{0}(z, u)<v\right\}$ and $\left\{v<-\rho_{1}(z, u)\right\}((z, u) \in \Omega)$ are pseudoconvex. The disc is contained in the domain $\rho_{0}(z, u)-M<v<-\rho_{1}(z, u)+M$, at first for large $M$ and by letting $M \rightarrow 0$, squeezed in the domain $\rho_{0}(z, u)<v<-\rho_{1}(z, u)$, and one proceeds as in [BG]. The constant $C$ is eventually determined by the gradient of $\rho_{0}$ and $\rho_{1}$ at $\left(z_{0}, u_{0}\right)$.

We now indicate how to obtain $\rho_{0}$; the function $\rho_{1}$ is done similarly. Let $\tilde{g}$ be a continuous function on $\mathbb{R}$ with the property that

$$
\tilde{g}(u)=\min _{(z, u) \in \partial \Omega} g(z, u),
$$

for all $u$ for which the right hand sides make sense. Notice that $\{(z, w): v>\tilde{g}(u)$, $(z, u) \in \partial \Omega\}$ is pseudoconvex. Choose balls $B_{1} \Subset B_{2} \Subset V$ about $\left(z_{0}, u_{0}\right)$. Let $g^{\prime}$ be a smooth function on $\bar{\Omega} \cap \bar{B}_{2}$ which is equal to $g$ on $B_{1} \cap \partial \Omega, \leq g$ on $B_{2} \cap \partial \Omega$ and $\leq \tilde{g}$ in a neighborhood of $\partial B_{2} \cap \bar{\Omega}$. Such a smooth function exists and on $V$ the $C^{2}$ norm may be bounded by a constant depending on the $C^{2}$ norm of $g$ on $V$ and the sup norm of $\tilde{g}$ on $\{u: \exists z$ such that $(z, u) \in V\}$. Replacing $\rho$ by $\{\exp (\lambda \rho)-1\} / \lambda, \lambda$ sufficiently large, and shrinking $B_{2}$ if necessary, we may assume that $\rho$ is strictly plurisubharmonic on a neighborhood of $\left(z_{0}, u_{0}\right)$ containing $\bar{B}_{2} \cap \Omega$. Now there exists a constant $A$ depending on the $C^{2}$ norm of $g^{\prime}$ and the Levi form of $\partial \Omega$ within $V$ only, such that $g^{\prime \prime}(z, u)=g^{\prime}(z, u)+A \rho(z, u)$ is strictly plurisubharmonic. Hence the domain $\left\{(z, w): v>g^{\prime \prime}(z, u),(z, u) \in\right.$ $\left.\partial \Omega \cap B_{2}\right\}$ is pseudoconvex. Now take

$$
\rho_{0}= \begin{cases}\tilde{g}, & \text { if }(z, u) \in \bar{\Omega} \cap B \backslash B_{2} \\ \max \left\{\tilde{g}, g^{\prime \prime}\right\} & \text { if }(z, u) \in \bar{\Omega} \cap B_{2} .\end{cases}
$$

This satisfies the requirements because $\tilde{g} \geq g^{\prime \prime}$ close to the boundary of $B_{2}$. 
Corollary 2.12. With the notation as above, suppose that $\Gamma$ is approximated by smooth graphs $\Gamma_{j}$ over smooth domains, the convergence being in $C^{2}$ norm in a neighborhood of $\left(z_{0}, u_{0}+i g\left(z_{0}, u_{0}\right)\right)$.

If $p_{j} \in \Gamma_{j}$ tend to $\left(z_{0}, u_{0}+i g\left(z_{0}, u_{0}\right)\right)$, then discs with boundary in $\Gamma_{j}$ will make an angle with the forbidden direction $\chi\left(z_{0}, u_{0}+i g\left(z_{0}, u_{0}\right)\right)$ of at least $C$ for $j$ large enough.

Proof. Observe that $\chi_{\Gamma_{j}}$ tends to $\chi_{\Gamma}$ at least in a neighborhood of $\left(z_{0}, u_{0}+i g\left(z_{0}, u_{0}\right)\right)$ and apply the previous Lemma.

We return to Kruzhilin's theorem for a moment. Choose an orientation of $\partial W$. Clearly, the level sets of $x_{3}$ and hence the boundaries of discs filling $S$ inherit this orientation. This gives a coorientation to the forbidden directions.

We will occasionally view a graph of a function $P(z, \bar{z})$ with domain $\mathcal{G} \subset \mathbb{C}$ as $\Gamma_{\operatorname{Im} P}$ on $\{u=\operatorname{Re} P: z \in \mathcal{G}\} \subset \mathbb{C} \times \mathbb{R}$. Now consider a part of $\Gamma$ given as a graph of $v=\operatorname{Im} P(z, \bar{z})$ over the boundary of the domain $u>\operatorname{Re} P(z, \bar{z}), z \in G \subset \mathbb{C}$, such that there are no $\mathrm{CR}$ singularities in this part of $\Gamma$. An easy computation gives that on such a part of $\Gamma$ the integral curves of $\chi$ are pulled back to solutions of the differential equation

$$
\frac{d z(t)}{d t}=z^{\prime}=\frac{\partial P(z, \bar{z})}{\partial \bar{z}} .
$$

This gives "forbidden curves" in $G \subset \mathbb{C}$ : A disc with boundary in $\Gamma$, part of which is given by $(z, f(z))$ on a domain $D_{f}$ never has $\partial D_{f}$ tangent to a forbidden curve.

Lemma 2.13. We keep the notation as above and assume that $\Gamma$ is approximated by smooth surfaces $\Gamma_{j}$ which are given as graphs of functions $P_{j}$ over $G$. Let $K$ be compact in $G$. There exist positive constants $c_{K}, d_{K}$ and a sector $S_{K}$ of the form $\left\{z:|\arg z|<c_{K},|z|<d_{K}\right\}$ or $-\left\{z:|\arg z|<c_{K},|z|<d_{K}\right\}$ such that for every $z_{0} \in G$ the following holds. Every analytic disc with boundary in $\Gamma$ or in approximating surfaces $\Gamma_{j}$ and passing through $\left(z_{0}, P\left(z_{0}, \bar{z}_{0}\right)\right)$, respectively $\left(z_{0}, P_{j}\left(z_{0}, \bar{z}_{0}\right)\right)$, can be written as a graph $(z, f(z)), z \in D_{f}$, such that the domain $D_{f}$ has the property

$$
z_{0}+\left(\frac{\partial P}{\partial \bar{z}}\left(z_{0}\right)\right) S_{K} \subset D_{f}
$$

Proof. Theorem 2.1 gives that any disc with boundary in $\Gamma$ is of the form $(z, f(z)), z \in D_{f} \subset G$.

If the boundary $\gamma$ of the disc passes through $\left(z_{0}, P\left(z_{0}, \bar{z}_{0}\right)\right)$, it follows from Lemma 2.11 that the boundary of $D_{f}$ meets the line $z_{0}+t(\partial P / \partial \bar{z})\left(z_{0}\right)$ under 
an angle at least $C$. Furthermore, the boundary of $D_{f}$ will not return to the forbidden curve passing through $z_{0}$ by Corollary 2.9. As $\partial P / \partial \bar{z}$ varies smoothly with $z$ on $G$, it follows that $D_{f}$ contains one of the domains $z_{0} \pm\left(\partial P / \partial \bar{z}\left(z_{0}\right)\right) S_{K}$. The coorientation forces one choice valid for all discs with boundary passing through $\left(z_{0}, P\left(z_{0}, \bar{z}_{0}\right)\right)$. Finally, application of Corollary 2.12 gives the result for neighboring surfaces.

Note that it is allowed that the surfaces $\Gamma_{j}$ are equal to $\Gamma$ above part of $U$.

3. Local polynomially convex hulls at CR singularities. In this section we will show that under suitable pseudoconvexity conditions, graphs of fairly smooth complex valued functions on domains in $\mathbb{C}$ have at isolated $\mathrm{CR}$ singularities of positive index a non-trivial local polynomially convex hull containing analytic discs. Contrary to the smooth index 1 situation, these discs may have their boundary passing through the CR singularity. This can already occur if the index is one and the graph is of class $C^{2-\varepsilon}, \varepsilon>0$. We will also find a condition which assures that the boundary passes around the CR singularity.

Theorem 3.1. Let $\varphi$ be a $C^{k}, k \geq 2$, smooth function on a disc in $\mathbb{C}$ about the origin. Suppose that $\varphi$ has an isolated CR-singularity at the origin of index $j, 0<j<k$. Denote the graph of $\varphi$ by $\Gamma_{\varphi}$. If $\operatorname{Re} \varphi(z, \bar{z}) / z^{j-1}$ is strictly subharmonic on a punctured neighborhood of 0 , then there exist analytic discs with boundary in $\Gamma_{\varphi}$. In case $j=1$, there exists $\varepsilon_{0}>0$ such that for $0<\varepsilon<\varepsilon_{0}$, $\left(\{|z|<\varepsilon\} \cap \Gamma_{\varphi}\right)^{\wedge} \backslash\{|z|<\varepsilon\} \cap \Gamma_{\varphi}$ is a Levi-flat hypersurface consisting of analytic discs with boundary in $\{|z|<\varepsilon\} \cap \Gamma_{\varphi}$.

Proof. First assume that the index of the origin is 1 . Let $\Omega=\{\operatorname{Re} \varphi(z, \bar{z})-$ $u<0\} \cap\left\{|z|^{2}+u^{2}<M\right\}$, where $M$ is so small that $\Omega$ is topologically a ball, which satisfies the conditions of Theorem 2.1 with $h^{+}(z)=\sqrt{M^{2}-|z|^{2}}$ and $h^{-}=\max \left\{\operatorname{Re} \varphi(z),-\sqrt{M^{2}-|z|^{2}}\right\}$ on the simply connected domain $D=\{|z|<$ $M\} \cap\left\{\sqrt{M^{2}-|z|^{2}}>\operatorname{Re} \varphi(z)\right\}$ (this is a condition on $M$ ). Note that $\operatorname{Im} \varphi$ is a well defined continuous function on $\bar{\Omega}$ and denote its graph over $\partial \Omega$ by $\Gamma$. Let the disc $\Delta=\Delta(0, \delta)$ be compactly contained in $D$, such that 0 is the only CRsingularity of $\varphi$ on $\Delta$. Let $U$ be open in $\mathbb{C} \times \mathbb{R}$, such that $U \cap \partial \Omega=\left\{\left(z, h^{-}(z)\right)\right.$ : $z \in \Delta\}$.

Now approximate $\Omega \times i \mathbb{R}$ from inside with strictly pseudoconvex domains $\Omega_{j} \times i \mathbb{R}$ such that $\Omega_{j}$ is smoothly bounded, and approximate $\varphi$ with a smooth function $g_{j}$ on $\partial \Omega_{j}$. In view of Thom's transversality Theorem [GG], this can be done in such a way that the graphs $\Gamma_{j}$ of $g_{j}$ on $\partial \Omega_{j}$ are spheres in the boundary of smooth strictly pseudoconvex domains, with only finitely many exceptional points. Notice that $\Gamma_{j}$ can be chosen to approximate $\Gamma$, at least in $C^{2}$ sense 
in compact sets where $\Gamma$ is $C^{2}$, hence we may assume that $\Gamma_{j}$ is totally real above any part of $U$ contained in $\varepsilon<|z|<\delta, 0<\varepsilon<\delta$ fixed. It may well happen that the approximating $\Gamma_{j}$ have several CR singularities elsewhere. For $\varepsilon<\varepsilon_{0}<\delta$ and $j$ sufficiently large, the curve $|z|=\varepsilon_{0}$ on $\Gamma_{j} \cap(U \times i \mathbb{R})$ has index 1 and bounds a simply connected domain in $\Gamma_{j}$. We apply Corollary 2.10 and Theorem 2.1 to obtain discs of the form $\left(z, f_{j}(z)\right), z \in D_{f_{j}}$ with boundary in $\Gamma_{j} \cap(U \times i \mathbb{R}) \cap\left\{|z| \leq \varepsilon_{0}\right\}$ passing around an elliptic point in $\Gamma_{j}$ close to the origin and touching $|z|=\varepsilon_{0}$. These discs form a normal family: The functions $f_{j}$ are bounded and the domains $D_{f_{j}} \subset\left\{|z|<\varepsilon_{0}\right\}$ (viewed as appropriate conformal maps from the unit disc) contain points $z$ with $|z|$ close to $\varepsilon_{0}$ and close to 0 , hence in view of Lemma 2.13 no subsequence can collapse to a set without interior. After taking subsequences if necessary, a limit function $f$ will exist on a domain $D_{f} \subset\left\{|z|<\varepsilon_{0}\right\}$.

The proof of Theorem 2.1, cf. [CSh] gives that $f$ extends to a function in $A\left(D_{f}\right)$ which defines an analytic disc with boundary in $\Gamma$, contained in the Leviflat hypersurface $\Gamma_{\Phi}$ bounded by $\Gamma$ given by Theorem 2.1. Since it is a limit of discs of index 1, its boundary must either pass around the origin or the origin is in its boundary. Moreover, $\max _{z \in D_{f}}|z|=\varepsilon_{0}$, hence the boundary of the disc is contained in $\Gamma_{f}$. We obtain such a disc for every $\varepsilon_{0}$ sufficiently small.

To finish the proof in the index 1 case, note that in the proof above we could have chosen any extension $g$ of $\operatorname{Im} \varphi$ from $\partial \Omega \cap U \cap\left\{|z| \leq \varepsilon_{0}\right\}$ to $\partial \Omega$ and would have obtained a Levi-flat hypersurface $\Gamma_{G}$ bounded by $\Gamma_{g}$, which contains the same $\operatorname{disc}\left(f, D_{f}\right)$ by uniqueness. Hence $\left(\{|z|<\varepsilon\} \cap \Gamma_{\varphi}\right)^{\wedge} \backslash\{|z|<\varepsilon\} \cap \Gamma_{\varphi}$ is contained in all of the hypersurfaces $\Gamma_{G}$ independent of the particular choice of the extension $g$. The intersection of these hypersurfaces contains analytic discs with boundary in $\{|z|<\varepsilon\} \cap \Gamma_{\varphi}$. Suppose it contains an other point. This would belong to a disc in some $\Gamma_{G}$ with part of the boundary outside $\{|z|<\varepsilon\} \cap \Gamma_{\varphi}$. Then for an appropriate $g^{\prime} \geq g$, Corollary 2.2 shows that the disc has no intersection with $\Gamma_{G^{\prime}} \backslash \Gamma_{g^{\prime}}$.

The index $>1$ case is treated as follows: We just have obtained a disc $(z, f(z)), z \in D_{f}$, with boundary in $\left\{w=\varphi(z, \bar{z}) / z^{j-1}\right\}$, so $\left(z, z^{j-1} f(z)\right), z \in D_{f}$, is a disc with boundary in $\{w=\varphi(z, \bar{z})\}$.

The subharmonicity or equivalently, the fact that the surface lies in the boundary of a pseudoconvex domain is rather essential for the proof to work. Compare, however, Corollary 3.4.

As an application we consider a parabolic point in a surface $\Gamma$. It is fairly easy to see that the index at such a point can only be $-1,0$ or $1,[\mathrm{~J}]$.

Corollary 3.2. Let 0 be a parabolic point of index 1 in a $C^{2}$ surface $\Gamma$ in $\mathbb{C}^{2}$. At 0 the local polynomially convex hull is formed by a Levi-flat hypersurface foliated by analytic discs with boundary in $\Gamma$. 
Proof. Changing coordinates if necessary, $\Gamma$ may be given as a graph of $|z|^{2}+\operatorname{Re} z^{2}+O\left(|z|^{3}\right)$, so that the conditions of the theorem are satisfied.

We do not know if the discs may have their boundaries passing through 0 in this situation.

Theorem 3.3. Let $F(z, \bar{z})$ be homogeneous of degree $k$ in $z$ and $\bar{z}$ and $C^{2}$ smooth away from the origin in $\mathbb{C}$. Suppose that 0 is an isolated CR-singularity of $\Gamma=\{w=F(z, \bar{z})\}$ and that the index at 0 equals $j, 0<j<k$. If $\operatorname{Re}\left(F / z^{(j-1)}\right)$ is a subharmonic but nowhere harmonic function, then $\Gamma$ is not locally polynomially convex at 0 . It will contain a $(2 j-1)$ parameter family of analytic discs with boundary in $\Gamma$ passing around 0 if and only if the curve $C$ in $\mathbb{C}$,

$$
C(z)=\frac{F(z, \bar{z})}{z^{k}}, \quad \text { defined on }|z|=1
$$

has the following property: If for two different points $z_{1} \neq z_{2}$ on the unit circle $C\left(z_{1}\right)=C\left(z_{2}\right)$, then $z_{1}$ and $z_{2}$ divide the unit circle in two segments of length at least $\pi /(k-j+1)$. Moreover, if the index $j>1$, then this family will fill an open neighborhood of 0 in $\mathbb{C}^{2}$. If this condition is not satisfied, then the hull contains at least a one parameter family of curves with boundary in $\Gamma$ passing through 0 .

Proof. We transform the problem to one with an index 1, isolated CRsingularity, by considering $\varphi(z, \bar{z})=F(z, \bar{z}) / z^{(j-1)}$. Let $\Gamma^{\prime}$ be the graph of $w=\varphi(z, \bar{z})$. Again $\Gamma^{\prime}$ is a graph over a subset of $\mathbb{C} \times \mathbb{R}$ :

$$
\Gamma^{\prime}=\{((z, u), v): v=\operatorname{Im} \varphi(z, \bar{z})\} \quad(\text { on } u=\operatorname{Re} \varphi(z, \bar{z})) .
$$

It follows from Theorem 3.1 that there exists an analytic disc with boundary in $\Gamma^{\prime}$ of the form $(z, f(z))$, with domain $G$ and $\max _{z \in \bar{G}}|z|=1$.

Suppose that $G$ contains a neighborhood of 0 , so that we have a disc with boundary not passing through 0 of index 1 and assume that the curve (3.1) has self-intersection. Replacing $\varphi$ by $\varphi(z)-c z^{k-j+1}$, which is just a change of coordinates, we may assume that $\varphi=0$ on two half-lines emanating from the origin and that $\operatorname{Re} \varphi$ equals 0 on the boundary of an open sector $S$ of width less than $\pi /(k-j+1)$. As $\operatorname{Re} \varphi$ is subharmonic, nowhere harmonic and homogeneous of degree $k-j+1$, the segments of the unit circle where $\operatorname{Re} \varphi$ is negative have length strictly less than $\pi /(k-j+1)$, while the segments where $\operatorname{Re} \varphi$ is positive have length strictly greater than $\pi /(k-j+1)$. Also each "negative" segment is bounded by "positive" segments. Proofs of these properties in case $\operatorname{Re} \varphi$ is real analytic on the unit circle are in $[\mathrm{BF}]$, Section 1 . They can be easily adapted to the $C^{2}$ case. As a consequence, $\operatorname{Re} \varphi$ is negative on $S$ 
Now form $\Omega$ as in the previous proof. Let $g$ be a continuous extension of $\operatorname{Im} \varphi$ from $\partial \Omega \cap\{u=\operatorname{Re} \varphi\}$ to $\partial \Omega$ with the property that $g=0$ on $S \times\{0\} \cap \partial \Omega$. This can be done because $\operatorname{Im} \varphi=0$ on $S \times\{0\} \cap(\partial \Omega \cap\{u=\operatorname{Re} \varphi\})$. As by the maximum principle $\operatorname{Re} f \geq \operatorname{Re} \varphi$ on $\bar{G}$, with equality precisely on $\partial G$, it is clear that $\operatorname{Re} f$ will have zeros on $G \cap S$. It would follow that the discs defined by the function $f$ on $G$ and the function 0 on $S$ would have non-empty intersection. This contradicts Corollary 2.3.

Next suppose that $G$ has boundary passing through 0 . Homogeneity allows us to form for $t>0$,

$$
f_{t}(z)=t^{(k-j+1)}\left(f\left(\frac{z}{t}\right)\right)
$$

on $t G$. This is again a disc with boundary in $\Gamma^{\prime}$. It follows from Corollary 2.3 that for $t<s, G_{t} \subset G_{s}$ and $\operatorname{Re} f_{t}<\operatorname{Re} f_{s}$ on $G_{t}$. Thus $G$ is star shaped with respect to the origin. We have that $f=\varphi$ on $\partial G$. Therefore, $|f(z)| \leq C|z|^{(k-j+1)}$ on $\partial G$. A simple harmonic measure argument gives

$$
f(z) \leq C|z|^{(k-j+1)}
$$

on $\bar{G}$. Consider $S=\bigcup_{t>0} G_{t}$. Observe that $\bar{S} \neq \mathbb{C}$, for $G_{t}$ would violate Lemma 2.13. Now the functions $f_{t}, t>s$, are bounded on $G_{s}$ in view of (3.2). It follows that there exists a limit $f_{0}$ on $S$ that satisfies

$$
f_{0}(z)=t^{(k-j+1)}\left(f_{0}\left(\frac{z}{t}\right)\right)
$$

on $S$. Then $f_{0}(z)=|z|^{(k-j+1)}(f(z /|z|))$, hence $f_{0}(z) / z^{(k-j+1)}$ is a bounded function on $S$ and constant on each halfline in $\partial S$, hence a constant. Therefore,

$$
f_{0}(z)=c z^{(k-j+1)}
$$

on $S$. Hence, also $\varphi(z, \bar{z})=c z^{(k-j+1)}$ on the boundary of $S$. This amounts to selfintersection of the curve (3.1). Without loss of generality we may assume $c=0$. Then $\operatorname{Re} f_{t}<0$ on $G_{t}$ and hence, $\operatorname{Re} \varphi<0$ on $\bigcup_{t} G_{t}=S$. By the properties stated in the beginning of the proof, the length of the segment determined by $S$ is strictly less than $\pi /(k-j+1)$. The proof is complete for the index 1 case.

If $j>1$, then discs in the original $\Gamma$ are again defined by $\left(z, z^{j-1} f_{t}(z)\right)$, $z \in G_{t}$. If 0 is an interior point of $G_{t}$, we have a disc with boundary in the totally real points of $\Gamma$ with index $j$. We may apply Forstneric's results, $[\mathrm{F}$, p. 6], summed up in Theorem 2.7, to see that these discs belong to a $2 j-1$ parameter family which fills an open set $O$. In fact, as for every $\zeta$ in the unit disc the mapping

$$
\mathbb{R}^{j} \times \mathbb{R}^{j-1} \rightarrow \mathbb{C}, \quad(a, b) \mapsto L(a, b)(\zeta) \zeta^{j-1}
$$


with

$$
L(a, b)(\zeta)=a_{0}+\sum_{k=1}^{j-1}\left(a_{k}-i b_{k}\right) \zeta^{k}+\left(a_{k}+i b_{k}\right) \zeta^{-k}
$$

is surjective, his proof $[\mathrm{F}$, p. 26] gives that each disc in the family is contained in $O$, hence so is the origin.

Examples given in Section 4 show that self-intersection of the curve (3.1) may occur.

Corollary 3.4. Keep the notation of the theorem but only assume that $\operatorname{Re}\left(F / z^{(j-1)}\right)$ is strictly subharmonic on an open sector containing points $z_{1}, z_{2}$ on the unit circle and a separating segment of length $<\pi /(k-j-1)$. If the curve (3.1) satisfies $C\left(z_{1}\right)=C\left(z_{2}\right)$, then there exists a one parameter family of analytic discs with boundary in $\Gamma$ passing through 0 .

Proof. We may assume $F\left(z_{1}\right)=F\left(z_{2}\right)=0$. Extend $F$ outside the sector $S$ determined by $z_{1}$ and $z_{2}$ to a homogeneous function so that $\operatorname{Re}\left(F / z^{(j-1)}\right)$ is strictly subharmonic and apply the previous theorem. Its proof shows that the domains of $D_{f}$ will belong to $S$.

Theorem 3.5. Suppose that $\varphi(z, \bar{z})=P_{k}(z, \bar{z})+O\left(|z|^{k+1}\right)$ is smooth of class $C^{k}$ on a disc $\Delta$ about 0 of radius $r$, where $P$ is a homogeneous polynomial of degree $k$. Suppose that $P$ has an isolated $C R$ singularity of index $j>0$ at 0 and satisfies the conditions of Theorem 3.3 and that the associated curve (3.1) has no self-intersection at neighbouring points. Then the polynomially convex hull of $\Gamma_{\varphi}$ contains a $2 j-1$ - parameter family of analytic discs, which contains an open ball around 0 if $j>1$.

Proof. It suffices to deal with the index 1 case. Higher indices can be treated as in Theorem 3.2 by dividing by $z^{j-1}$. The expansion of the resulting function will start with a homogeneous function of degree at least 2 , and will be $C^{2}$ smooth on a punctured neighborhood of 0 . Thus the assumptions are that $\Gamma_{\varphi}$ has an index $1 \mathrm{CR}$-singularity at 0 and that the real part of $P_{k}$ is strictly subharmonic away from 0 . Let $\Gamma$ be the graph of $\varphi$ and $\Gamma_{0}$ be the graph of $P_{k}$.

Consider the functions $\varphi_{t}(z)=t^{-k} \varphi(t z)$ with graph $\Gamma_{t}$. These functions are well defined on $\Delta$ and converge to $P_{k}$. The convergence is in $C^{2}$ norm on any compact set of $\Delta$ not containing 0 , i.e., compact sets above not containing $0, \Gamma_{t}$ will be $C^{2}$ close to $\Gamma_{0}$. As the polynomially convex hull of $\Gamma_{0}$ contains analytic discs with boundary in $\Gamma_{0}$ passing around 0 , the same is true for $\Gamma_{t}$ for $t$ sufficiently close to 0 , by Theorem 2.7 . However, if $(z, f(z)), z \in D_{f}$ and 0 contained in $D_{f}$, is a disc with boundary in $\Gamma_{t}$, then $\left(z, t^{k} f(z / t)\right)$ is a disc with boundary in $\Gamma$. 


\section{Remarks 3.6.}

1. The proofs of Theorems 3.1, 3.3 and 3.5 also work if we assume that $\operatorname{Re} \varphi(z, \bar{z}) / z^{\ell-1}$ is strictly subharmonic for some $\ell$ less then the index. From Forstneric's theorem and the fact that the local polynomial hull of $w=$ $\varphi(z, \bar{z}) / z^{\ell-1}$ is a hypersurface it would follow that the discs have their boundaries passing through the origin. However, we could not find examples of such functions.

2. One might hope to define the local polynomially convex hull of a compact set $K$ at $p \in K$ as the germ at $p$ of a set $X$ with property that, for every $\varepsilon_{1}>0$, there is a $\varepsilon_{2}>0$ such that

$$
K \hat{\cap} B_{\varepsilon_{1}} \cap B_{\varepsilon_{2}}=X \cap B_{\varepsilon_{2}} .
$$

Here $B_{\varepsilon}$ denotes the closed ball around $p$ with radius $\varepsilon$. However, even for a smooth surface $\Gamma$ this kind of hull may not exist. This is the case at a CR singularity $p$ if $\widehat{K \cap B}_{\varepsilon}$ consists of shrinking discs with boundaries passing through $p$.

3. Recent work with Jöricke shows that the self-intersection property of the curve (3.1) is equivalent with the presence of elliptic and hyperbolic sectors at the critical point at 0 of the vectorfield $\partial F / \partial \bar{z}$. In view of (2.1), hyperbolic sectors at a critical point of this vectorfield prevent the existence of small analytic discs with boundary passing around the critical point.

4. Examples. The degree 2 models being completely understood, we consider more complicated surfaces:

$$
\Gamma=\{w=P(z, \bar{z})\},
$$

where $P$ is a homogeneous polynomial of degree 3 in $z$ and $\bar{z}$. After changing coordinates, we may assume that $P$ is of the form

$$
P(z, \bar{z})=a z^{2} \bar{z}+b z \bar{z}^{2}+c \bar{z}^{3} .
$$

The index $I$ of $\Gamma$ at 0 equals the winding number of $\partial P / \partial \bar{z}$ over the unit circle. Thus

$$
\left.\frac{\partial P}{\partial \bar{z}}\right|_{|z|=1}=\left.\left(a z^{2}+2 b|z|^{2}+3 c \bar{z}^{2}\right)\right|_{|z|=1}=\left.\frac{1}{z^{2}}\left(a z^{4}+2 b z^{2}+3 c\right)\right|_{|z|=1} .
$$

Let $\zeta_{1}, \zeta_{2}$ be the zeros of $a \zeta^{2}+2 b \zeta+3 c$, with $\left|\zeta_{1}\right| \leq\left|\zeta_{2}\right|$. We find that

$$
I= \begin{cases}2 & \text { if }\left|\zeta_{2}\right|<1 \\ -2 & \text { if }\left|\zeta_{1}\right|>1 \\ 0 & \text { if }\left|\zeta_{1}\right|<1,\left|\zeta_{2}\right|>2 .\end{cases}
$$


If one of the $\left|\zeta_{j}\right|=1$, then the origin is a non-isolated CR-singularity.

We consider the index 2 case. Then we may assume that $a=1$, hence $-2 b=\zeta_{1}+\zeta_{2}, 3 c=\zeta_{1} \zeta_{2}$. Thus $|c|<\frac{1}{3}$ and an easy computation gives that

$$
\operatorname{Re}\left(\frac{P(z, \bar{z})}{z}\right)=\operatorname{Re}\left(|z|^{2}+b \bar{z}^{2}+c \frac{\bar{z}^{3}}{z}\right)
$$

is strictly subharmonic on $\mathbb{C} \backslash\{0\}$. We are in the situation of Theorem 3.3 and wish to choose $b, c$ in such a way that the analytic discs have their boundary passing through 0 . Thus the associated curve

$$
C(\vartheta)=e^{-2 i \vartheta}+b e^{-4 i \vartheta}+c e^{-6 i \vartheta}
$$

has to have self-intersection for values $\vartheta_{1}, \vartheta_{2}$ very close to each other. It is reasonable to view $C$ as a perturbation of a curve $\tilde{C}$ with $\tilde{C}^{\prime}\left(\vartheta_{0}\right)=0$ for some $\vartheta_{0}$, which we may assume to be 0 . Observe that

$$
C^{\prime}(\vartheta)=-\left.2 i e^{-i \vartheta}\left(\frac{\partial(P(z, \bar{z}) / z)}{\partial \bar{z}}\right)\right|_{z=e^{i \vartheta}} .
$$

Thus at least one of the $\zeta^{\prime}$ 's has to be close to 1 . We take $\zeta_{1}=\zeta_{2}=\frac{19}{20}$.

Example 4.1. The surface $\Gamma_{1}$ given by

$$
w=z^{2} \bar{z}-\frac{19}{20} z \bar{z}^{2}+\frac{361}{1200} \bar{z}^{3}
$$

has for every neighborhood $U$ of 0 a 1-parameter family of analytic discs with boundary in the surface and passing through 0 , contained in $U$.

Indeed, the associated curve becomes

$$
C(\vartheta)=\cos \vartheta-\frac{19}{20} \cos 2 \vartheta+\frac{361}{1200} \cos 3 \vartheta+i\left(\sin \vartheta-\frac{19}{20} \sin 2 \vartheta+\frac{361}{1200} \sin 3 \vartheta\right) .
$$

As the real part of $C$ is even and the imaginary part is odd, a zero of $\operatorname{Im} C$ at $0<\vartheta_{1}<\pi$ gives self-intersection of $C$ for $\vartheta_{1}$ and $\vartheta_{2}=-\vartheta_{1}$. Writing down a Taylor series for $\operatorname{Im} C$ we find

$$
\frac{1}{400} \vartheta-\frac{203}{800} \vartheta^{3}+\frac{1}{5 !}\left(1-\frac{19}{20} 2^{5}+\frac{361}{1200} 3^{5}\right) \vartheta^{5}-\frac{1}{7 !}\left(\left(1-\frac{19}{20} 2^{7}+\frac{361}{1200} 3^{7}\right) \vartheta^{7}+\cdots .\right.
$$

This is easily seen to be positive for $\vartheta$ very close to 0 and negative at $\vartheta=\frac{1}{5}$; there is a zero on $\left[0, \frac{1}{5}\right]$, which is in fact approximately $\frac{1}{10}$. As the distance $\left|\vartheta_{1}-\vartheta_{2}\right|<\frac{2}{5}<\pi / 2$, we have discs with boundary passing through 0 according to Theorem 3.3. 
It is easy to see that if we take $b$ and $c$ relatively small in (4.1), e.g., $\mid 1+b+$ $c|>| b+c|+| c \mid$, this self-intersection doesn't occur and we obtain a 3 parameter family of analytic discs in the hull which fills an open neighborhood of 0 according to Theorem 3.3.

Next, we give an example of a surface with an isolated CR-singularity of index 1 that has a hull made up of analytic discs with boundary passing through 0 . The method of finding the example is as in Example 4.1, but things are complicated because the subharmonicity of the real part is no longer automatic. Moreover, for later purposes we want no terms of type $\bar{z}^{k}, z \bar{z}^{k-1}$ present.

Finding the next example was greatly facilitated, if not made possible, by using Maple. In fact, the previous example was found with help of Maple too.

Example 4.2. The surface $\Gamma_{2}$ defined by

$$
w=\frac{1}{2} z^{6} \bar{z}^{2}-\frac{34}{25} z^{5} \bar{z}^{3}+\frac{63031}{40000} z^{4} \bar{z}^{4}-\frac{436491}{500000} z^{3} \bar{z}^{5}+\frac{761211}{4000000} z^{2} \bar{z}^{6}
$$

has an isolated CR-singularity of index 1 at the origin. Its local polynomially convex hull consists of a 1-parameter family of analytic discs with boundary in $\Gamma_{2}$ and passing through 0 .

We proceed as in the previous example, starting off with

$$
P(z, \bar{z})=a_{2} z^{2} \bar{z}^{6}+a_{3} z^{3} \bar{z}^{5}+a_{4} z^{4} \bar{z}^{4}+a_{5} z^{5} \bar{z}^{3}+a_{6} z^{6} \bar{z}^{2} .
$$

We compute $\partial P / \partial \bar{z}$; and substitute $\bar{z}=1 / z$. The result is of the form

$$
\frac{Q\left(z^{2}\right)}{z^{3}}
$$

where $Q$ is a polynomial of degree 4 . The surface has index 1 at 0 if and only if $Q$ has two roots of norm $<1$ and two of norm $>1$. Motivated by the previous example, we try $Q$ a perturbation of $(\zeta-1)^{4}$ and compute $P$ accordingly. Here some experiments with Maple had to be done. The following choice for $Q$ works

$$
Q(\zeta)=\left(\zeta-\frac{99}{100}\right)^{2}\left(\zeta-\left(\frac{21}{20}+\frac{i}{4}\right)\right)\left(\zeta-\left(\frac{21}{20}-\frac{i}{4}\right)\right)
$$

This yields the polynomial in (4.2). Again the real part of the associated curve $C(\vartheta)$ is seen to be even, while the imaginary part is odd. Taylor expansion can be used to show that $\operatorname{Im} C(\vartheta)$ has a zero at approximately .0187 , so that selfintersection of $C$ occurs for $\vartheta_{1}, \vartheta_{2}$ which differ less than $\frac{1}{25}(<\pi / 8)$. We omit the details. 
Forstneric and Stout $[\mathrm{FS}]$ showed that a totally real surface $\Gamma$ with an isolated hyperbolic CR singularity $q$ in $\mathbb{C}^{2}$ is locally polynomially convex at $q$. In fact, they showed that in a neighborhood of $q$, the analytic polynomials are dense in the continuous functions on $\Gamma$. Forstneric [F2] has an example of a smooth locally holomorphically convex surface in $\mathbb{C}^{2}$ with an isolated CR-singularity of index 0 at which it admits a discrete family of analytic discs having their boundaries arbitrarily close to and passing around the singularity. Our next example shows that the Forstneric-Stout result can not be extended to polynomial surfaces with an isolated degenerated CR singularity of negative index, and complements the Forstneric example in that the surface is the graph of a homogeneous polynomial and the local hull is a hypersurface in $\mathbb{C}^{2}$.

Example 4.3. The surface $\Gamma_{3}$ defined by

$$
w=\frac{1}{2} z^{4} \bar{z}^{2}-\frac{34}{25} z^{3} \bar{z}^{3}+\frac{63031}{40000} z^{2} \bar{z}^{4}-\frac{436491}{500000} z \bar{z}^{5}+\frac{761211}{4000000} \bar{z}^{6}
$$

has an isolated CR-singularity of index -1 at the origin. Its local polynomially convex hull consists of a 1-parameter family of analytic discs with boundary in $\Gamma$ and passing through 0 .

The defining polynomial of (4.3) is obtained by dividing the polynomial of (4.2) by $z^{2}$. Thus it is clear that $\Gamma_{3}$ is a surface with a CR-singularity of index -1 at the origin. Now suppose that $(z, f(z)), z \in D \subset \mathbb{C}$ is an analytic disc in $\Gamma_{2}$. Then $0 \in \partial D$ and $f(z)=O\left(z^{8}\right)$ at 0 , because of (3.2). Therefore, $f(z) / z^{2}$ is analytic on $D$ and continuous on $\bar{D}$. We conclude that the one-parameter family of discs $\left(z, f_{t}(z)\right)$ close to the origin in the surface $\Gamma_{2}$ gives rise to a one parameter family $\left(z, f_{t}(z) / z^{2}\right)$ close to the origin in $\Gamma_{3}$.

More precisely, let $\varepsilon>0, B(0, \varepsilon)$ the closed ball with radius $\varepsilon$ in $\mathbb{C}^{2}$ and consider the polynomial map from $\mathbb{C}^{2}$ to $\mathbb{C}^{2}$ :

$$
F:(z, w) \mapsto\left(z, z^{2} w\right),
$$

We have $F^{-1}\left(\Gamma_{2}\right)=\Gamma_{3} \cup\{z=0\}$. We claim that $K=\left(\Gamma_{3} \cap F^{-1}(B(0, \varepsilon))\right)^{\wedge}$ consists precisely of the discs $\left(z, f_{t}(z) / z^{2}\right)$, where $\left(z, f_{t}(z)\right)$ are the discs forming the hull of $\Gamma_{2} \cap B(0, \varepsilon)$. Indeed, if $F\left(z_{0}, w_{0}\right) \notin\left(\Gamma_{2} \cap B(0, \varepsilon)\right)^{\wedge}$, then $\left(z_{0}, w_{0}\right) \notin K$, by considering polynomials $P \circ F$. If $F\left(z_{0}, w_{0}\right)$ is in one of the discs $\left(z, f_{t}(z)\right)$ in the hull of $\Gamma_{2} \cap B(0, \varepsilon)$, then $\left(z_{0}, w_{0}\right)$ belongs to the disc $\left(z, f_{t}(z) / z^{2}\right)$. Finally, the remaining case is that $\left(z_{0}, w_{0}\right)$ is mapped onto $\Gamma_{2}$, but doesn't belong to $\Gamma_{3}$. This means that $z_{0}=0, w_{0} \neq 0$. Such points can not belong to $K$, because at a point of the form $\left(0, w_{0}\right) \in K$ with $\left|w_{0}\right|=\max _{\{(0, w) \in K\}}|w|$ the function $\left(w+w_{0}\right) / 2$ would obtain a local maximum outside the Shilov boundary, which contradicts the local maximum modulus principle, cf. [W]. 
Acknowledgment. It is my pleasure to thank Prof. N. G. Kruzhilin, who suggested Theorem 3.1 and its proof resulting in a much more transparent paper. Also I am indebted to the Mittag-Leffler Institute for hospitality. I started working on this topic when I visited during the special year in complex analysis $1987-1988$.

\section{REFERENCES}

[BG] E. Bedford \& B. GAVEAU, Envelopes of holomorphy of certain 2-spheres in $\mathbb{C}^{2}$, Amer. J. Math. 105 (1983), 975-1009.

[BF] E. BEDFORD \& J. E. FornæSS, A construction of peak functions on weakly pseudoconvex domains, Ann. of Math. 107 (1978), 555-568.

[BK] E. BEDFORD $\&$ W. KLINGEnBERG JR., On the envelope of holomorphy of a 2-sphere in $\mathbb{C}^{2}$, J. Amer. Math. Soc. 4 (1991), 623-646.

[B] E. BISHOP, Differentiable manifolds in complex Euclidean space, Duke Math. J. 32 (1965), 1-21.

[CSh] E. M. ChIRKA \& N. V.ShCherbina, Pseudoconvexity of rigid domains and foliations of hulls of graphs, Ann. Scuola Norm. Sup. (to appear).

[F1] F. ForstnerIC, Analytic disks with boundaries in a maximal real submanifold of $\mathbb{C}^{2}$, Ann. Inst. Fourier 37 (1987), 1-44.

[F2] A smooth holomorphically convex disc in $\mathbb{C}^{2}$ that is not locally polynomially convex, Proc. Amer. Math. Soc. 116 (1992), 411-415.

[FS] F. Forstneric \& E. L. Stout, A new class of polynomially convex sets, Arkiv för Mat. 29 (1991), 51-62.

[G] J.GLobevNiK, Perturbation by analytic discs along maximal real submanifolds of $\mathbb{C}^{n}$, Math. Z. 217 (1994), 287-317.

[GG] M. Golubitsky \& V.Gulllemin, Stable Mappings and Their Singularities, Springer-Verlag, New York, 1973.

[H] G. A. HARRIS, Degenerate surfaces in $\mathbb{C}^{2}$, Proc. Sympos. Pure Math. 52 (1991), 179-189.

[HT] D. C. Hill \& G. TAIANA, Families of analytic disks in $\mathbb{C}^{n}$ with boundaries in a prescribed CR manifold, Ann. Scuola Norm. Sup. Pisa 5 (1978), 327-380.

[J] B. JÖRICKE, Oral communication.

[KW] C. KENIG 6 S. WEBSTER, The local hull of holomorphy of a surface in the space of two complex variables, Invent. Math. 67 (1982), 1-21.

[K] N. G. KRUZHILIN, Two-dimensional spheres in the boundaries of strictly pseudoconvex domains in $\mathbb{C}^{2}$, Math. USSR Izvestiya 39 (1992), 1151-1187.

[M] V. P. MASLOV, Théorie des Perturbations et Méthodes Asymptotiques, translated from the Russian, Dunod Gauthiers-Villars, Paris, 1972.

[Sh] N. V. ShCherbina, On the polynomial hull of a graph, Indiana Univ. Math. J. 42 (1993), 477-503.

[W] J. Wermer, Banach Algebras and Several Complex Variables, Springer-Verlag, New York, 1976.

Faculteit Wiskunde en Informatica

Universiteit van Amsterdam

Plantage Muidergracht 24

1018 TV Amsterdam, The Netherlands

E-MAIL: janwieg@fwi.uva.nl 